ORIGINAL STUDY

\title{
Drug-induced sleep endoscopy - decisional factor in obstructive sleep apnea treatment
}

\author{
Nicoleta Dumitrescu ${ }^{1,2}$, Codrut Sarafoleanu ${ }^{1,2}$ \\ ${ }^{1}$ ENT\&HNS Department, "Sfanta Maria" Hospital, Bucharest, Romania \\ 2"Carol Davila" University of Medicine and Pharmacy, Bucharest, Romania
}

\section{ABSTRACT}

BACKGROUND. Drug-Induced Sleep Endoscopy (DISE) is a sleep apnea diagnostic procedure which allows direct view the obstruction while inducing sleep using analgosedation with propofol. Many studies highlighted the importance of DISE in finding the level of obstruction and choosing the specific treatment.

MATERIAL AND METHODS. We performed DISE under propofol sedation in 27 patients diagnosed with sleep apnea who addressed to our clinic for further investigation and treatment. We assessed the obstruction type and severity using the Fujita scale, VOTE (velum, oropharynx, tongue base, epiglottis) and NOHL (nose, oropharynx, hypopharynx, larynx). Finally, we correlated the DISE findings with the polysomnographic results.

RESULTS. We observed significant correlations between the obstruction severity seen at DISE and the polysomnographic results. Further, we could decide the appropriate treatment for our patients, whether surgical or not (continuous positive airway pressure - CPAP).

CONCLUSION. Sleep endoscopy represents a very important method in establishing the topographic diagnostic of sleep apnea patients, being useful for the therapeutic decision and the postoperative assessment. We recommend drug-induced sleep endoscopy as elective investigation regarding the diagnostic and treatment of sleep apnea patients.

KEYWORDS: sleep apnea, sleep endoscopy.

\section{INTRODUCTION}

It is well known the implication of Obstructive Sleep Apnea Syndrome (OSAS) in daytime fatigue, changes in personality and diminished working capacity, as well as in developing cardiovascular diseases and more and more numerous traffic accidents ${ }^{1,2}$. That is why the correct diagnostic is of crucial importance and the assessment procedure must provide a complete clinical history and a thorough evaluation of the anatomical regions of the upper airway ${ }^{3}$.

The polysomnographic study is considered to be the gold standard diagnostic test for sleep apnea, offering complete information about the OSAS severity, the patient's clinical status and the presence of possible complications ${ }^{4}$. Because of the repeated failure of surgery and low CPAP compliance, the Drug-Induced Sleep Endoscopy (DISE) technique has been developed as an additional diagnostic tool for polysomnography and has permitted an adapted therapeutical strategy for OSAS patients. In the course of time, it became a widespread diagnostic method for the upper airway evaluation, preferred both by patients and doctors for its short duration and clear outcome.

Even though in 2014 the European position paper on drug-induced sedation endoscopy reached consensus in several aspects regarding the DISE procedure ${ }^{5}$, a recent survey made by Veer et al. showed that there is still need for a better patient selection, a sedation protocol and a universally accepted scoring system ${ }^{6}$.

Therefore, the objective of our study was to assess the patient characteristics, polysomnographic results and DISE practice, in order to increase patient compliance, reduce time until diagnostic and aim a better treatment outcome.

\section{MATERIAL AND METHODS}

We performed a one-year prospective study including a total of 27 patients diagnosed with SOAS. We in- 
cluded in the study adult patients diagnosed with sleep disorders and no contraindication for surgery or for mandibular advancement device fitting. We excluded patients with morbid obesity.

The study design implied a primary clinical and paraclinical evaluation followed by a "tailor-made" surgical intervention (septoplasty, turbinoplasty, uvulopalatoplasty, pillar implants, tongue base resection, partial epiglottectomy) or a non-surgical treatment (mandibular advancement device - MAD; continuous positive airway pressure - CPAP) and reevaluation after 3 months. First, we performed a clinical evaluation and a detailed anamnesis of both patient and his/her partner asking for consent for participating in the study and evaluating his/her quality of life using the visual analogue scale (VAS), the Epworth Scale and the Pittsburgh scale. The clinical assessment was measured using the Body Mass Index (BMI), the Mallampati score and the Friedman classification. The diagnostic assessment was composed of polysomnography (PSG) with or without CPAP titration and Drug-Induced Sleep Endoscopy (DISE) under propofol sedation $(1.5 \mathrm{mg} / \mathrm{kg})$. The polysomnographic results consisted of Apnea-Hypopnea Index (AHI), Snore Flags Index (SFI) and Desaturation Index (Desat index). The sleep endoscopy findings were classified using the Fujita classification, VOTE (Velum, Oropharynx, Tongue Base, Epiglottis) and NOHL (Nose, Oropharynx, Hypopharynx, Larynx) represented by: 0 no obstruction or vibration $(<50 \%) ; 1$-partial obstruction or vibration $(50-75 \%) ; 2$ - complete obstruction (> 75\%); $\mathrm{X}$ - cannot be viewed. Each DISE examination lasted between 10 to 20 minutes while we recorded and analysed the anatomic changes that caused the symptoms of sleep apnea and whether there was a single site or multiple sites of obstruction. We repeated the same protocol after 3 months.

We assessed the results using Microsoft Office Excel Software and we performed the statistical analysis by conducting the Two-sample t-test for means comparison.

\section{RESULTS}

After applying the inclusion and exclusion criteria, a total of 27 patients were admitted in our study, $33.33 \%$ female and $66.67 \%$ male (female:male $=9: 18$ ), with the mean age of 47.3 and a mean Body Mass Index (BMI) of 28.2. They presented for low quality of life as the Visual Analogue Scale showed and the mean VAS was 4.4. Using the Pittsburgh questionnaire, we identified the distribution of sleep quality in the group: $44.44 \%$ of patients $(n=12)$ were satisfied with their sleep, $37.04 \%(n=10)$ declared a poor quality of sleep and $18.52 \%(\mathrm{n}=5)$ were experiencing an unrestful sleep (Figure 1).

Further, we performed a polysomnographic examination that revealed a reversed proportion to the subjective responses. We diagnosed $55.6 \%$ of patients $(n=15)$ with severe OSAS (mean AHI $=41.91$ events/hour), 37\% $(\mathrm{n}=10)$ with moderate OSAS (mean AHI $=20.66$ events/ hour) and $7.4 \%(n=2)$ with mild OSAS (mean AHI $=12.10$ events/hour).

In our group, $88.89 \%$ of patients $(n=24)$ presented multilevel obstruction and the most frequent combination we observed was vellum-oropharynx. The complete obstruction (type 2) was present mostly in vellum and tongue base levels. Regarding the type, the anteroposterior one registered by far the highest number of complete obstructions. The partial narrowing (type 1) was evenly distributed among the airway passage, except for the nasal region where we found the most insignificant obstruction (Figure 2).

The Drug-Induced Sleep Endoscopy showed a total number of 31 complete obstructions (type 2) from which $61.3 \%$ were anteroposterior, $25.8 \%$ were lateral and $12.9 \%$ were concentric types. Regarding the partial obstructions (type 1), we found a total number of 22, including $36.36 \%$ anteroposterior, $31.82 \%$ lateral and $31.82 \%$ concentric types (Figure 3).

Further, looking at each level of the upper airway, 33 complete and 36 partial obstructions resulted. Among the complete obstructions, we observed $33.34 \%$ at the vellum, $24.24 \%$ at the tongue base, $21.21 \%$ at the oropharynx, $15.15 \%$ at the epiglottis and $6.06 \%$ at the nasal levels. As for the partial obstructions, we have noted $25 \%$ at the vellum, $22.22 \%$ at the tongue base, $27.78 \%$ at the oropharynx, $19.44 \%$ at the epiglottis and $5.56 \%$ at the nasal levels (Figure 4).

We summarized the PSG and DISE results in two tables representing the AHI values, depending on the level and the type of obstruction and also on their degrees according to the VOTE classification. As expected, the highest AHI scores were observed during complete obstruction (mean $\mathrm{AHI}=34.84$ /hour). Regarding the level of obstruction, we observed more apnea episodes when patients developed vellum (mean $\mathrm{AHI}=33.10$ /hour) and epiglottis narrowing (mean AHI = 33.73/hour) (Table 1). All our patients presented anteroposterior collapse at different levels, but the lateral collapse had a greater impact on PSG results (mean $\mathrm{AHI}=33.13$ /hour) (Table 2).

We repeated the polysomnography before and 3 months after the surgical procedure and we observed an almost $30 \%$ decrease in the severe OSAS group and almost $20 \%$ increase in the mild OSAS group. Therefore, we may conclude that the adapted treatment, whether surgical or not, was efficient and provided the expected outcome (Figure 5).

We compared the polysomnographic parameters (represented by AHI) and the sleep endoscopy findings (quantified by the VOTE classification) at the moment of presentation and 3 months after treatment. Using the Twosample t-test, we observed a significant decrease in both measurements indicated by the p-value lower than 0.05 . This similar behaviour of the AHI and VOTE points that polysomnographic and sleep endoscopy results may indi- 


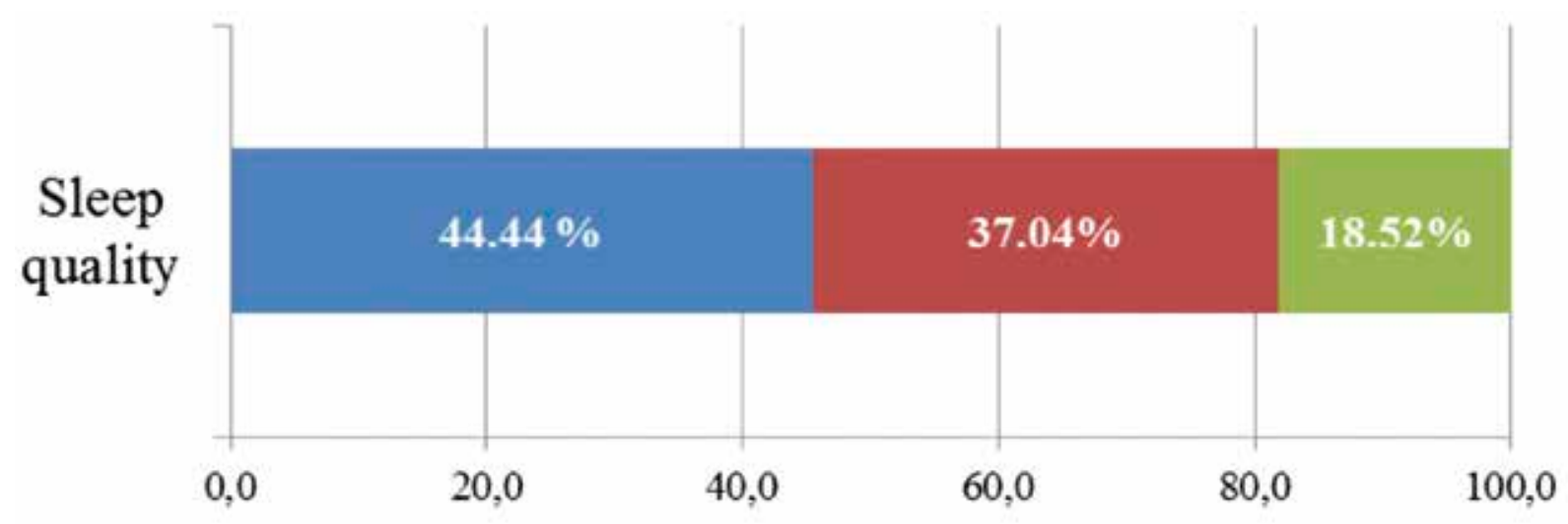

Good $=$ Low $\equiv$ Very low

Figure 1 Distribution of sleep quality resulting from the Pittsburgh questionnaire.

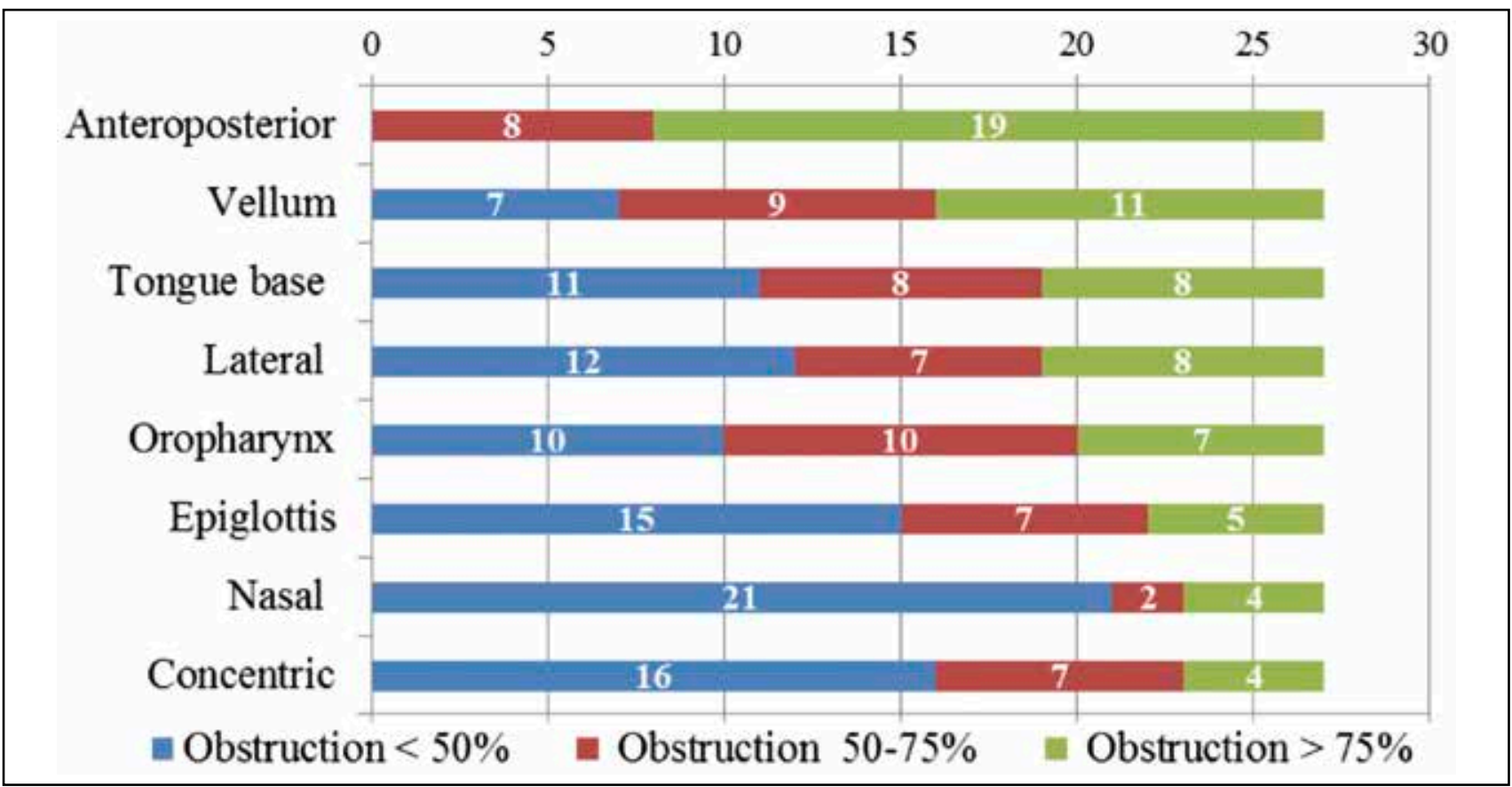

Figure 2 Obstruction severity distributed by type and level within our group.

cate the same SOAS severity (Figure 6).

In addition, we provide representative images collected during the sleep endoscopy conducted for our patients (Figures 7-11). We compared open airway images (a) with different types of obstruction (b).

\section{CASE REPORT}

We present the case of a 45-year-old female patient who was referred to our clinic for daytime sleepiness (Epworth
Score of 10), headache and high blood pressure. The clinical evaluation revealed BMI of $25.1 \mathrm{~kg} / \mathrm{m}^{2}$ and blood pressure of $150 / 85 \mathrm{mmHg}$. A year before presentation our patient underwent an uvulopalatoplasty intervention with pillar implants, but with no significant improvement (Figure 12). During this period, she continued to use CPAP with poor compliance. The polysomnographic evaluation with CPAP titration reported: $\mathrm{AHI}=22.9 / \mathrm{h} ; \mathrm{SFI}=90.5 / \mathrm{h}$; average $\mathrm{SpO}_{2}=95 \%$; lowest $\mathrm{SpO}_{2}=86 \%$; CPAP pressure of $14 \mathrm{cmH}_{2} \mathrm{O}$.

Furthermore, we performed a Drug-Induced Sleep En- 


\section{Complete obstruction}

\section{Partial obstruction}

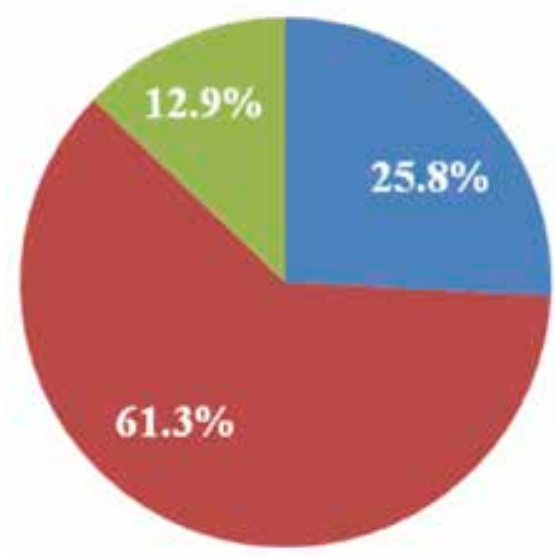

= Lateral

$31.82 \%$

$31.82 \%$

= Anteroposterior

w Concentric

\section{$61.3 \%$}

Complete obstruction

Partial obstruction
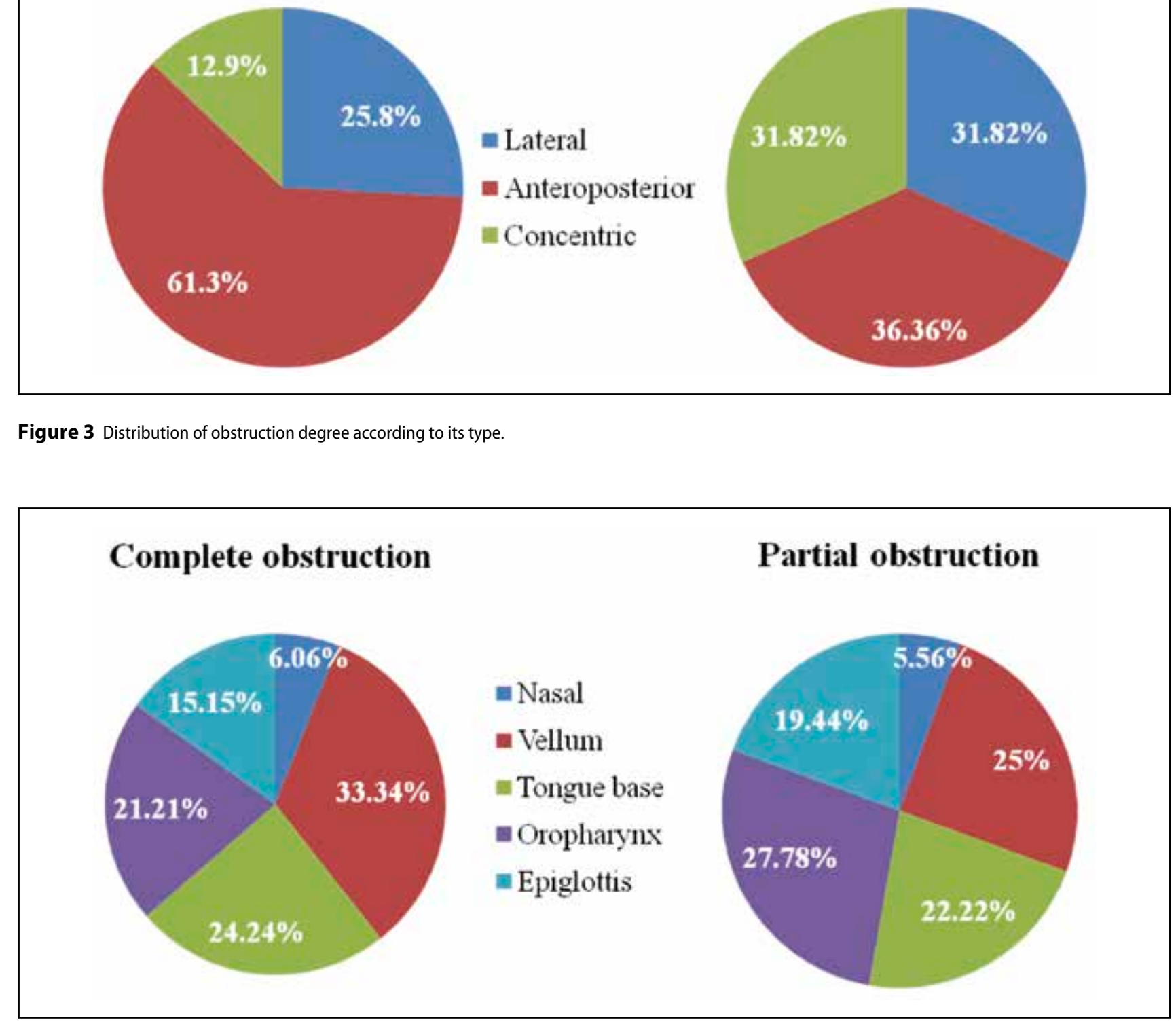

Figure 3 Distribution of obstruction degree according to its type.

Figure 4 Distribution of obstruction degree according to its level.

\section{Table 1}

Variation of Apnea-Hypopnea Index (AHI) depending on the obstruction level and severity.

\begin{tabular}{cccc}
\hline Obstruction level & & AHI & \multicolumn{1}{c}{} \\
\hline & 0 & 1 & 21.45 \\
\hline Nasal - & 32.70 & 43.5 & 41.2 \\
\hline Vellum & 34.2 & 23.9 & 34.5 \\
\hline Oropharynx & 30.3 & 30.6 & 35.6 \\
\hline Tongue base & 28.8 & 32.2 & 36.7 \\
\hline Epiglottis & 36.4 & 28.1 & \\
\hline
\end{tabular}




\section{Table 2}

Variation of Apnea-Hypopnea Index (AHI) depending on the obstruction type and severity.

\begin{tabular}{cccc}
\hline Obstruction type & & AHI & \\
\hline & 0 & 1 & 2 \\
\hline Lateral & 25.2 & 36.2 & 38 \\
\hline Anteroposterior & - & 29.5 & 37.3 \\
\hline Concentric & 25.9 & 33.6 & 34 \\
\hline
\end{tabular}

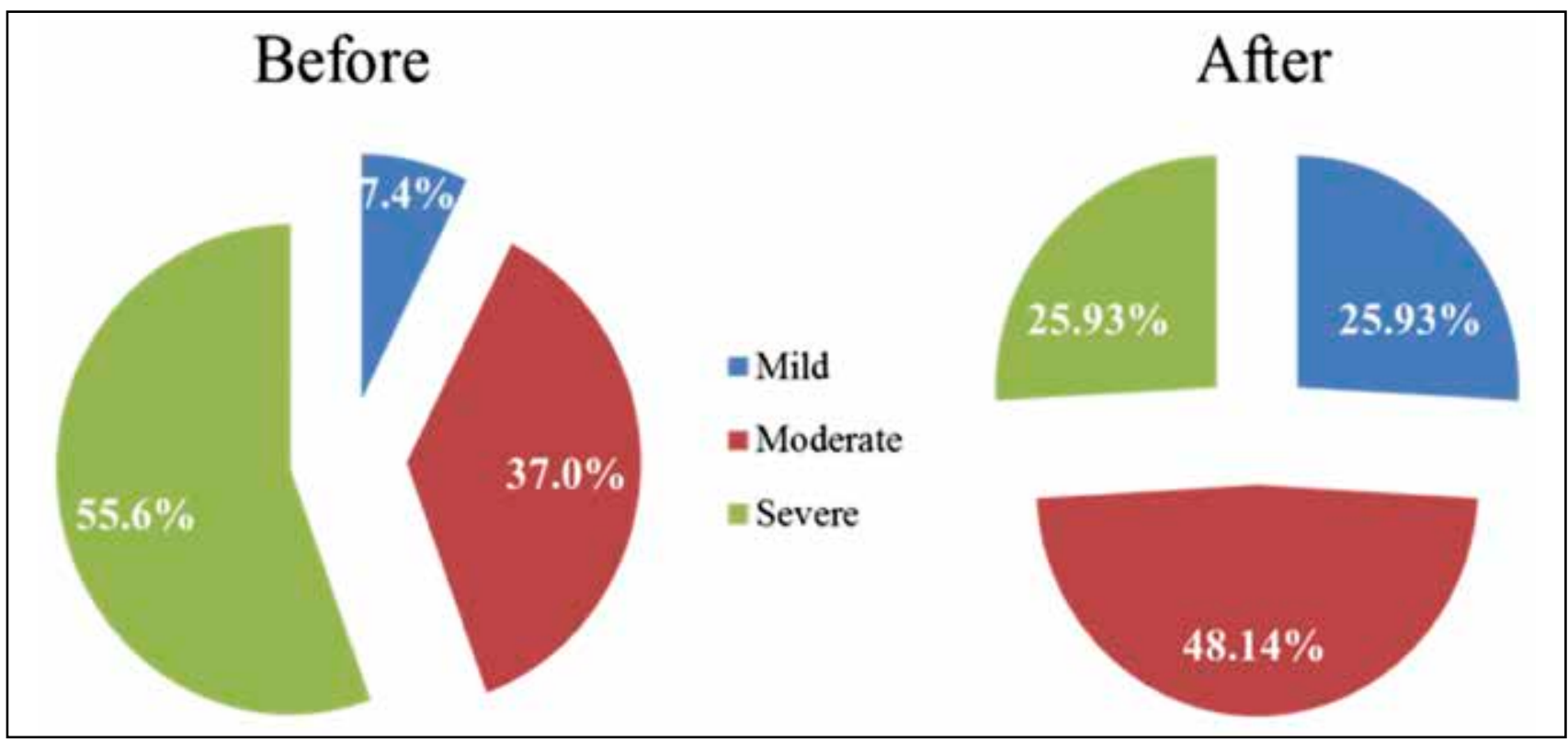

Figure 5 Distribution of sleep apnea severity before and 3 months after the tailored surgical procedure.

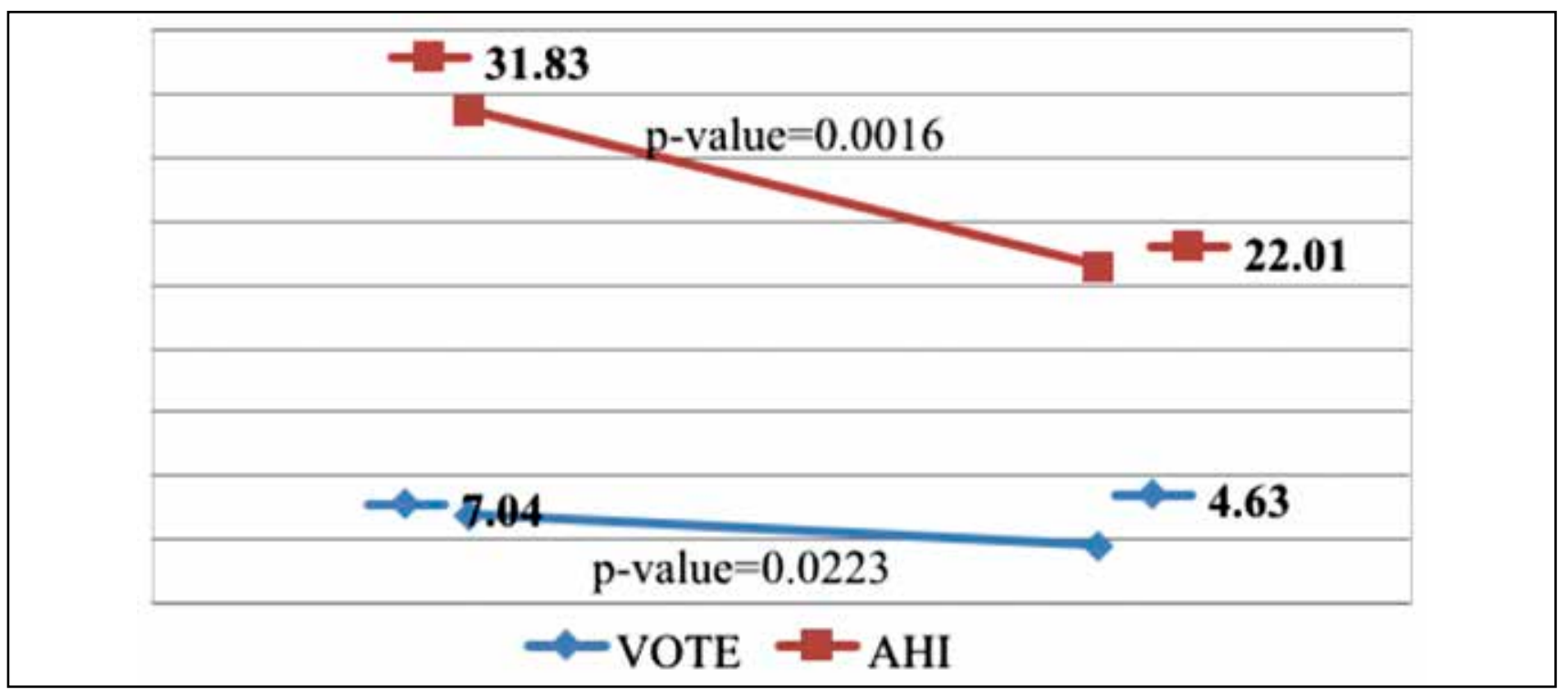

Figure 6 Statistically significant decrease (tested at 95\% confidence level) in measurements for polysomnography (AHI) and sleep endoscopy (VOTE classification) 3 months after surgery. of sleep apnea severity before and 3 months after the tailored surgical procedure. 

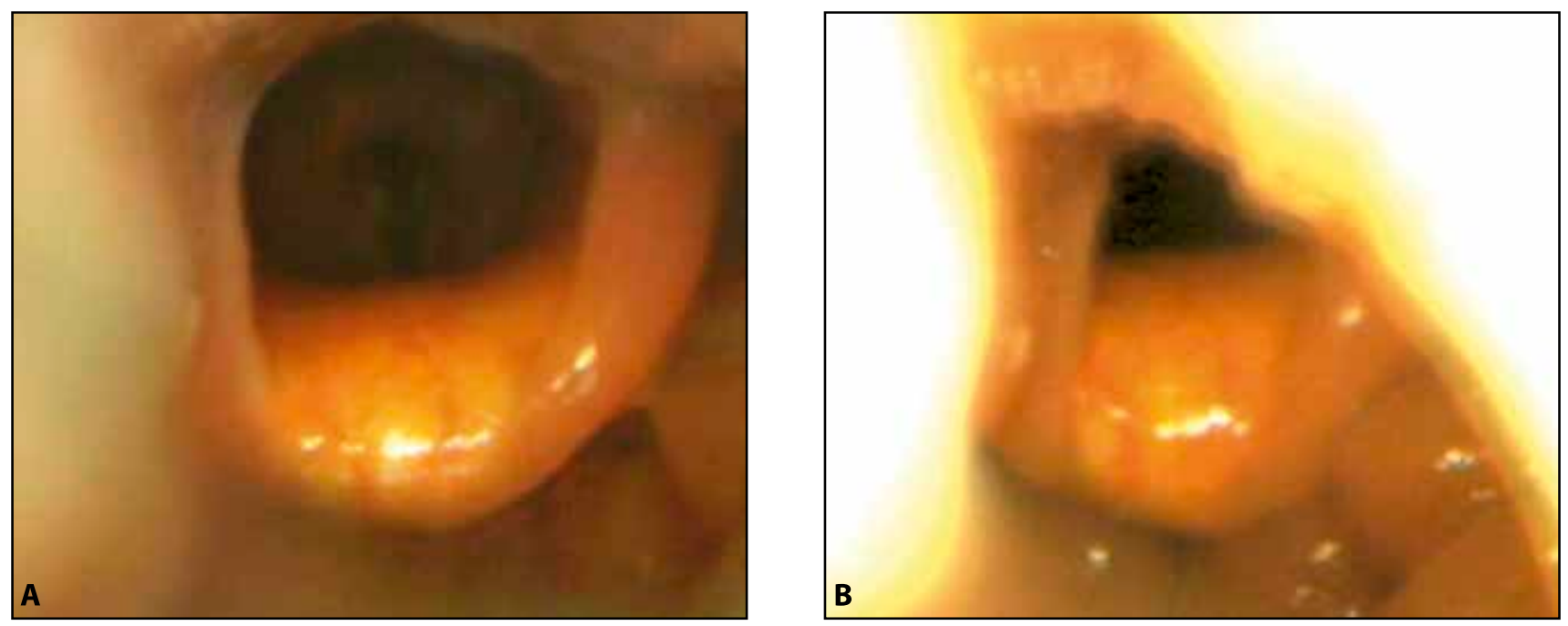

Figure 7 Hypopharynx: (a) Open airway. (b) Concentric obstruction.
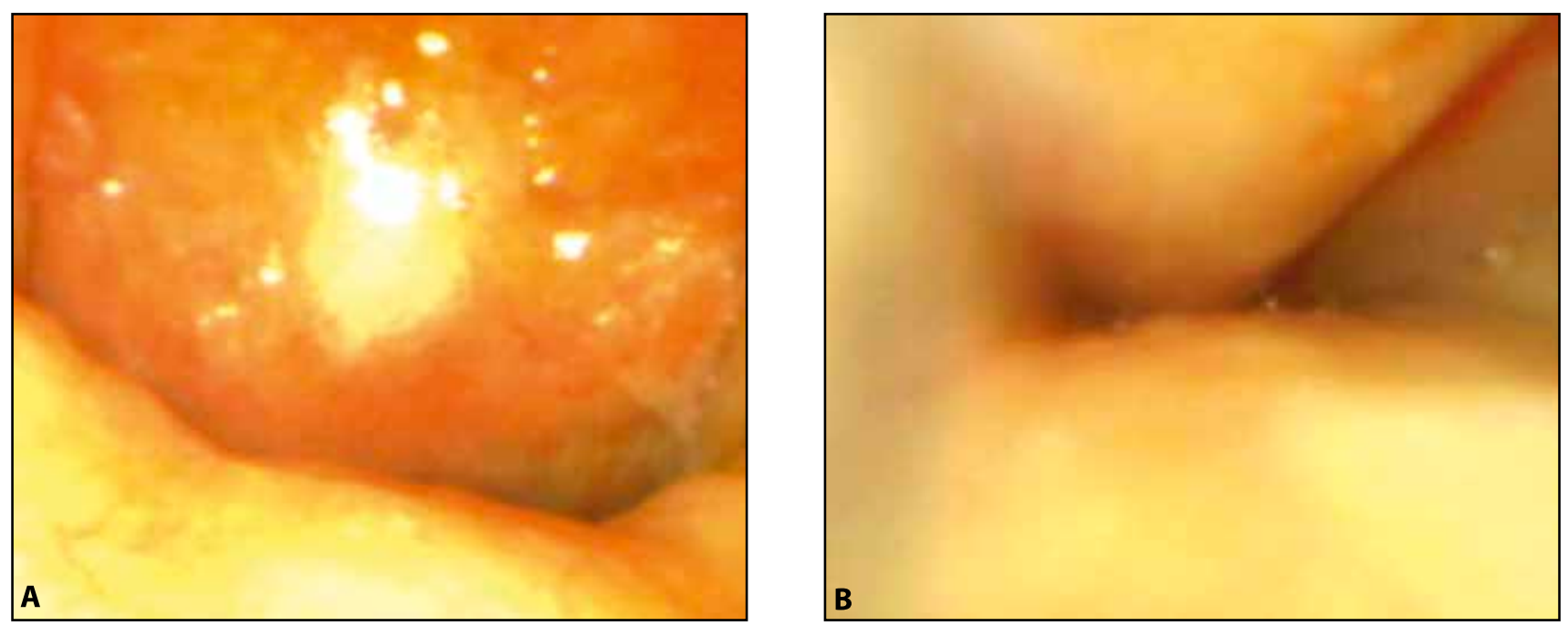

Figure 8 Vellum: (a) Open airway. (b) Concentric obstruction.
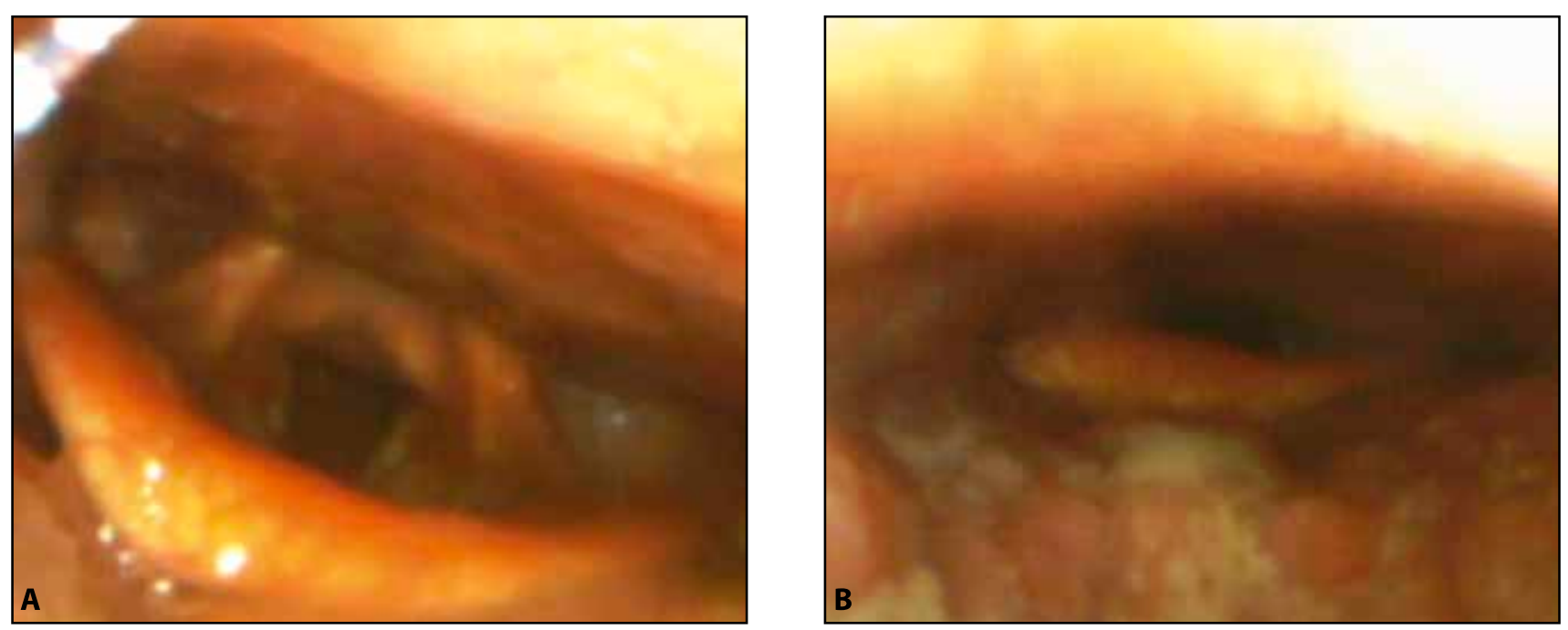

Figure 9 Hypopharynx: (a) Open airway. (b) Anteroposterior obstruction. 

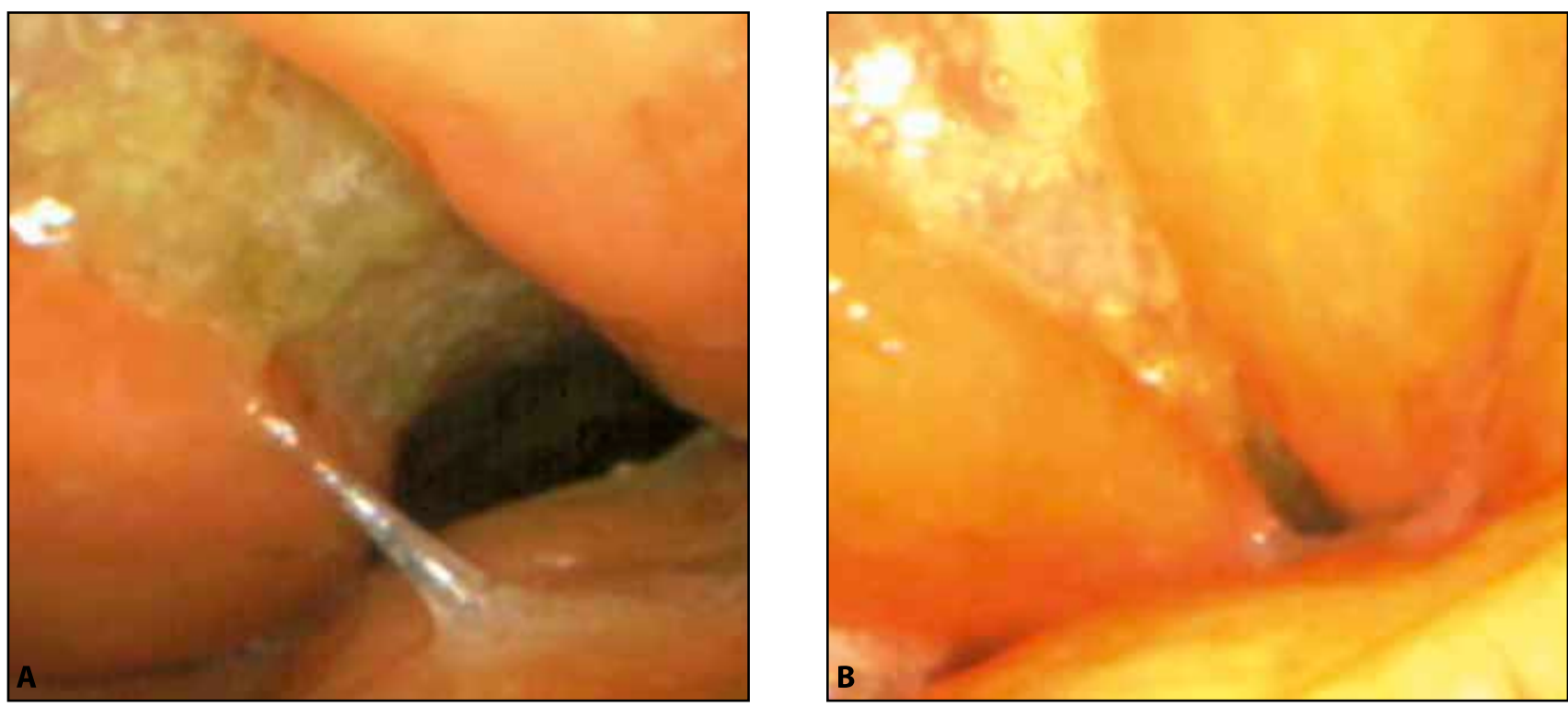

Figure 10 Hypertrophic posterior tonsillar pillars: (a) Open airway. (b) Lateral obstruction.
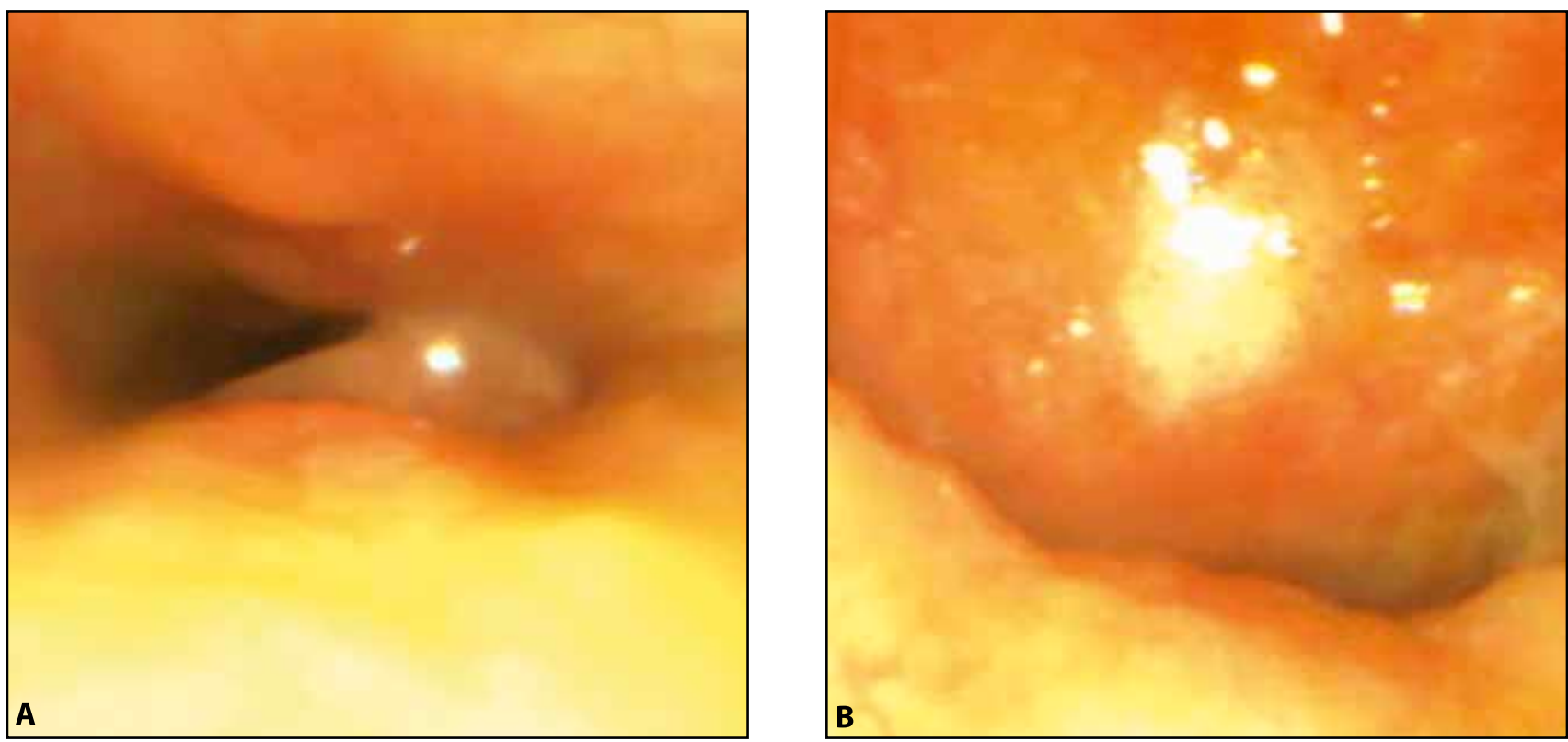

Figure 11 Pre (a) and postoperative (b) aspect of an anteroposterior obstruction due to soft palate hypotonia.

doscopy under propofol sedation, with progressive intravenous administration until a total of $100 \mathrm{mg}$ propofol. We noticed a hypertrophic tongue base with complete anteroposterior obstruction of the hypopharynx. When we manually advanced the mandible we observed a large widening of the air passage (Figure 13).

This finding lead us to conclude that the use of a mandibular advancement device should be the proper treatment for our patient. After 3 months we repeated the polysomnographic evaluation, while wearing the mandibular appliance, which showed significant improvement in the parameters $\left(\mathrm{AHI}=9 / \mathrm{h} ; \mathrm{SFI}=7.4 / \mathrm{h}\right.$; average $\mathrm{SpO}_{2}=95 \%$; lowest $\mathrm{SpO}_{2}=88 \%$ ) and no further need for CPAP use.

\section{DISCUSSIONS}

The technique of Sleep Endoscopy was first described in 1978 by Borowiecki in the naturally asleep patient, but only in 1991 did Croft and Pringle introduced the pharmacologic induction of sedation for flexible endoscopy in order to visualize the upper airway obstruction and vibra- 

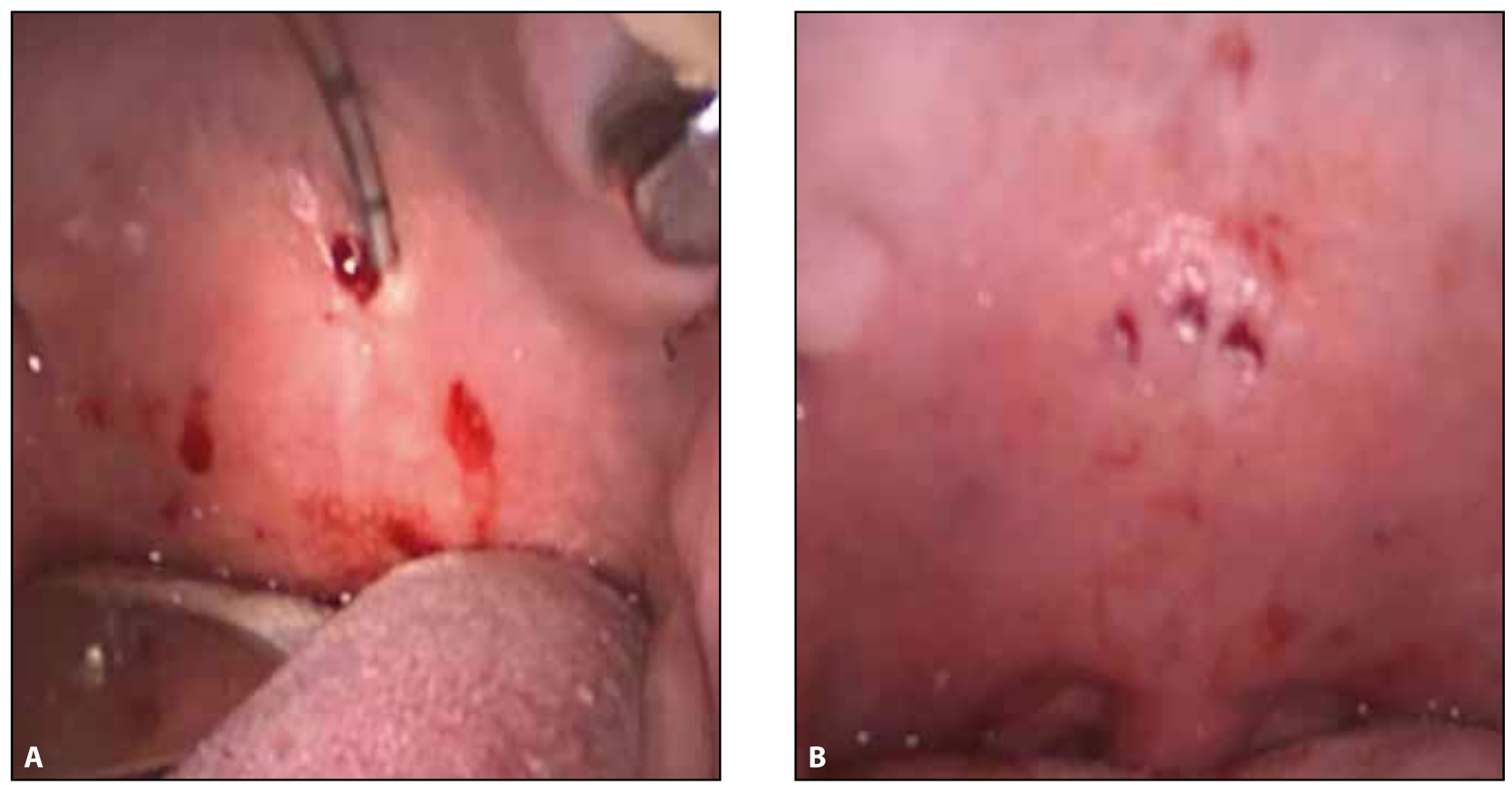

Figure 12 (a) Pillar implants intervention. (b) Postoperatory aspect.
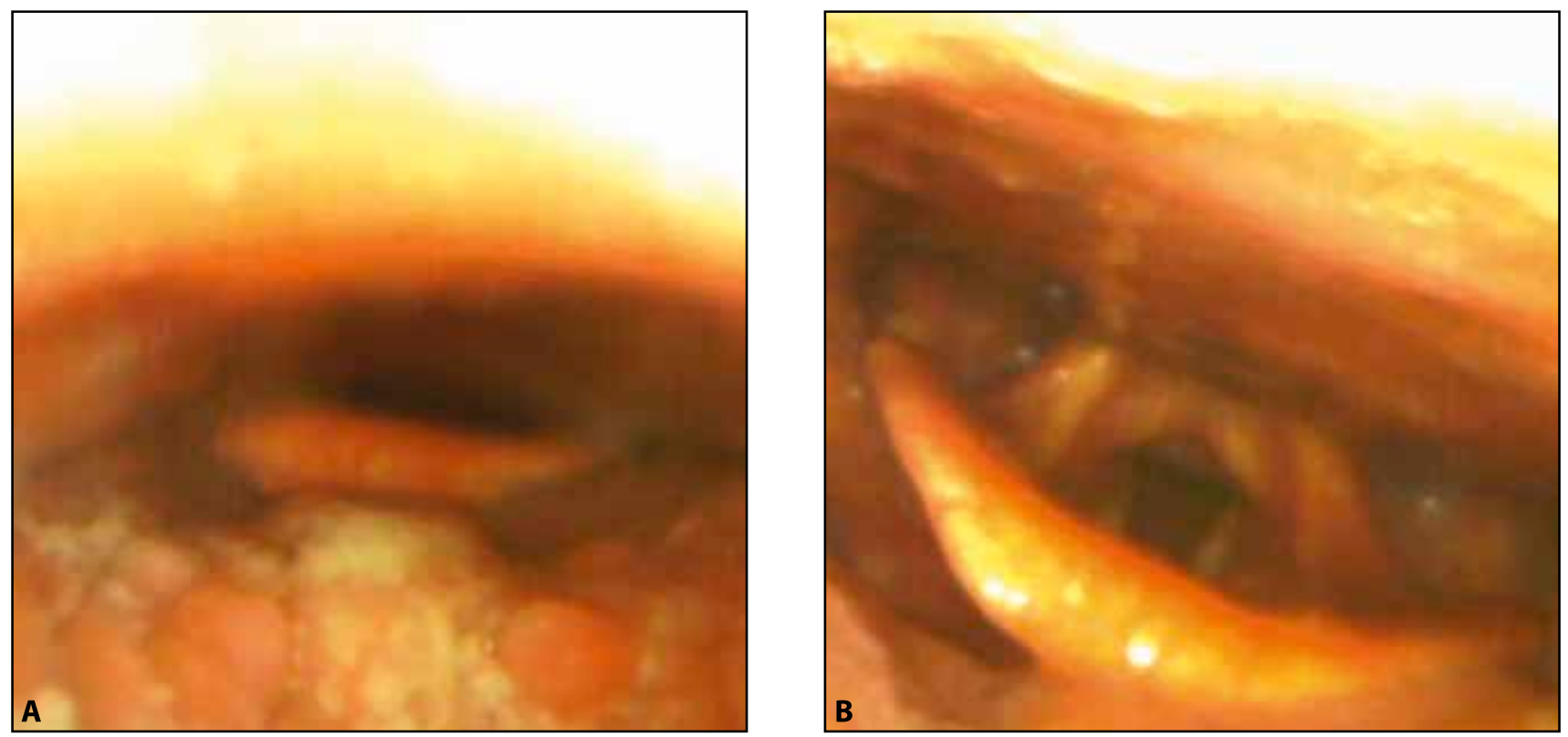

Figure 13 Drug-Induced Sleep Endoscopy: (a) Complete anteroposterior obstruction of the hypopharynx. (b) Mandibular advancement manoeuvre.

tion. It implies direct view of the nasal cavity, the pharynx, the tonsils, the tongue base, the epiglottis and the larynx using a flexible endoscope during analgosedation ${ }^{7,8}$.

Sleep is induced by intravenous progressive administration of propofol or midazolam until no response to verbal stimulation, further allowing the examination of the patient in almost similar conditions as those of the normal sleep. The flexible endoscope is introduced at the level of the nasal cavity after applying local anaesthetic (lidocaine $10 \%)$. The examination takes place in the clinic if the patient has a good health status (ASA score I or II) and AHI $<30 / \mathrm{h}$ in supine position or in the operation room if the patient has a higher ASA score or severe OSA ${ }^{9,10}$. The absolute contraindications of sedation are ASA score $\geq 3$ and propofol or midazolam allergies. The relative contraindications are severe OSA (AHI > 70/h) and severe obesity ${ }^{11}$. 
The examination may last 10 to 30 minutes, while the level and the severity of obstruction, if present, can be observed and certain manoeuvres, like chin lift, jaw thrust and head rotation, can be performed.

There are several types of classification used in the literature to identify and quantify the parameters of DISE, of which we mention the Fujita classification, VOTE (Velum, Oropharynx, Tongue Base, Epiglottis) and NOHL (Nose, Oropharynx, Hypopharynx, Larynx). The airway obstruction severity is graded as none - 0 ( $0-50 \%$ obstruction $)$, partial -1 (50-75\% obstruction) or complete -2 ( $>75 \%$ obstruction). The classifications may be applied during DISE and repeated while performing different manoeuvres ${ }^{12}$.

Finally, one may take the decision whether a surgical or non-surgical treatment could be recommended and, if so, at what level should one intervene.

\section{CONCLUSIONS}

Sleep endoscopy is a reliable method for the topographic diagnostic of sleep apnea patients. It has also a great impact upon the therapeutical decision and the postoperative assessment of the patients.

We recommend Drug-Induced Sleep Endoscopy as the examination of choice in sleep apnea patients. Further, we may ask ourselves if sleep endoscopy could replace polysomnography in the future.

Conflict of interest: The authors have no conflict of interest.

Contribution of authors: All authors have equally contributed to this work.

\section{REFERENCES}

1. Shamsuzzaman AS, Gersh BJ, Somers VK. Obstructive sleep apnea: implications for cardiac and vascular disease. JAMA. 2003;290(14):1906-14.

2. George CF, Smiley A. Sleep apnea and automobile crashes. Sleep. 1999;22(6):790-5.

3. Passali FM, Marzetti A, Bellussi L, Passali D. The diagnosis of obstructive sleep apnea syndrome. Romanian Journal of Rhinology. 2013;3(12):195-202.

4. Peverini F, Passali FM, Passali D. The role of polysomnography in OSAS patients. Romanian Journal of Rhinology. 2013;3(12):203-12.

5. De Vito A, Carrasco Llatas M, Vanni A, Bosi M, Braghiroli A, Campanini A, et al. European position paper on drug-induced sedation endoscopy (DISE). Sleep Breath. 2014;18(3):453-65. DOI: 10.1007/s11325-0140989-6. Epub 2014 May 26.

6. Veer V, Zhang H, Beyers J, Vanderveken O, Kotecha B. The use of druginduced sleep endoscopy in England and Belgium. Eur Arch Otorhinolaryngol. 2018;275(5):1335-42. DOI: 10.1007/s00405-018-4939-y. Epub 2018 Mar 19.

7. Borowiecki B, Pollak CP, Weitzman ED, Rakoff S, Imperato J. Fibrooptic study of pharyngeal airway during sleep in patients with hypersomnia obstructive sleep-apnea syndrome. Laryngoscope. 1978;88(8 Pt 1):1310-3

8. Croft CB, Pringle M. Sleep nasendoscopy: a technique of assessment in snoring and obstructive sleep apnoea. Clin Otolaryngol Allied Sci. 1991;16(5):504-9.

9. Hessel NS, de Vries N. Diagnostic work-up of socially unacceptable snoring. II. Sleep endoscopy. Eur Arch Otorhinolaryngol. 2002;259(3):158-61.

10. den Herder C, van Tinteren H, de Vries N. Sleep endoscopy versus modified Mallampati score in sleep apnea and snoring. Laryngoscope. 2005;115(4):735-9.

11. Hohenhorst W, Ravesloot MJL, Kezirian EJ, de Vries N. Drug induced sleep endoscopy in adults with sleep-disordered breathing: technique and the VOTE classification system. Oper Tech Otolaryngol Head Neck Surg. 2012;23(1):11-8. DOI: 10.1016/j.otot.2011.06.001.

12. Koutsourelakis I, Safiruddin F, Ravesloot M, Zakynthinos S, de Vries N. Surgery for obstructive sleep apnea: sleep endoscopy determinants of outcome. Laryngoscope. 2012;122(11):2587-91. DOI: 10.1002/ lary.23462. Epub 2012 Aug 1.v 